\title{
VARIATION OF THE MILNOR FIBRATION \\ IN PENCILS OF HYPERSURFACE SINGULARITIES
}

\author{
CLÉMENT CAUBEL
}

[Received 29 February 2000; revised 24 August 2000]

\section{Introduction}

Let $n$ be a positive integer and $f:\left(\mathbb{C}^{n+1}, \mathbf{0}\right) \rightarrow(\mathbb{C}, 0)$ the germ of a holomorphic function. If $\bar{B}_{\varepsilon}^{2 n+2}$ and $\bar{D}_{\eta}^{*}$ denote the closed ball in $\mathbb{C}^{n+1}$ with centre $\mathbf{0}$ and radius $\varepsilon$, and the punctured closed disc in $\mathbb{C}$ of centre 0 and radius $\eta$, respectively, the restriction of a representative of $f$ defined by

$$
f_{\varepsilon, \eta}: \bar{B}_{\varepsilon}^{2 n+2} \cap f^{-1}\left(\bar{D}_{\eta}^{*}\right) \stackrel{f}{\longrightarrow} \bar{D}_{\eta}^{*},
$$

with $0<\eta \ll \varepsilon \ll 1$, is a locally trivial fibration, which does not depend on the choice of $\varepsilon$ and $\eta$ provided they are sufficiently small. This is the Milnor fibration of $f$ (see [21]). It is the main object one considers when studying the topology of the holomorphic germ $f$.

The aim of this paper is to describe how the Milnor fibration varies in certain 1-parameter linear deformations, that is, those defined by

$$
f_{t}=f+t g
$$

where $g:\left(\mathbb{C}^{n+1}, \mathbf{0}\right) \rightarrow(\mathbb{C}, 0)$ is another germ of an analytic function having no common factor with $f$. In the case where $f$ has an isolated singular point at $\mathbf{0}$, Parusiński showed recently in [23] that, for these deformations, the constancy of the Milnor number $\mu\left(f_{t}\right)=\operatorname{dim}_{\mathbb{C}} \mathbb{C}\{\mathbf{z}\} /\left(\partial f_{t} / \partial \mathbf{z}\right)$ implies that all these germs have homeomorphic Milnor fibrations. This has to be compared with the paper of Lê and Ramanujam [13], which gives the same result for any deformation of a germ with an isolated singular point, but with the hypothesis $n \neq 2$.

When the critical locus Crit $f$ of $f$ is 1-dimensional, Iomdine in [8] and Lê in [11] introduced the so-called Iomdine-Le deformations of the type $f_{t}=f+t \ell^{N}$, with $\ell$ a generic linear form with respect to $f$ and $N \in \mathbb{N}, N \gg 1$. Using different methods, they showed that the Milnor fibre of $f$ embeds in that of $f_{t}$, and computed the difference between their Euler characteristics in terms of local invariants of the restriction of the pair $(f, \ell)$ to the critical locus of $f$. Siersma in [25] extended these results by first showing that the whole Milnor fibration of $f$ embeds in that of $f_{t}$ and then computing the difference between their monodromies. Shortly afterwards, Schrauwen in [24, Chapter 6] and Némethi in [22] established analogous results for a more general choice of $g$, but with the same assumption on the dimension of Crit $f$. Finally, Tibăr in [27] proved the embedding result of Siersma for Iomdine-Lê deformations without any restriction on the dimension. 
The proofs of all these statements (starting with the ones of Iomdine and Lê) are essentially based on the lifting in $\left(\mathbb{C}^{n+1}, \mathbf{0}\right)$ of isotopies in $\left(\mathbb{C}^{2}, \mathbf{0}\right)$ by means of the map $(f, g)$. The most extensive use of this technique was made by Lê in his work on the Cerf diagram (see [10] for instance). Other results based on this idea are the various decompositions of the Milnor fibre obtained first by Lê and Perron in [12], then by Vannier in [28], Massey in [19] and Tibăr in [26].

The first part of our work (see $\S 2$ ) is devoted to a unifying detailed description of the isotopies in $\left(\mathbb{C}^{2}, \mathbf{0}\right)$ which are the basis of all these results. They are maximal isotopies between subdiscs of complex lines transverse to a fixed plane curve germ. Under the map $(f, g)$, such a subdisc corresponds to a Milnor subfibre for the germ $f_{t}$, when $t$ is related to the slope of the complex line, the curve being the discriminant $\operatorname{Disc}(f, g)$.

Next (see $\S 3$ ), we impose on the pair $(f, g)$ a genericity condition: having no blowing up in codimension 0 (see Definition 3.1 and Example 3.3). This condition is in many ways minimal to get results like those stated above. We will say 'with no blowing up' in the sequel for convenience. The concept was introduced in [7] as the first requirement for a morphism $\Psi$ to admit a stratification satisfying Thom's $a_{\Psi}$-condition. We then prove the statements needed to get isotopies in $\left(\mathbb{C}^{n+1}, \mathbf{0}\right)$ from those constructed in the preceding section. One point should be mentioned here: in order to study the Milnor fibration of each germ $f_{t}$ by means of the map $(f, g)$, one must actually replace the ball $\bar{B}_{\varepsilon}^{2 n+2}$ in its definition by its intersection with the preimage by $(f, g)$ of a closed ball $\bar{B}_{\delta}^{4}$ around $\mathbf{0}$ in $\mathbb{C}^{2}$, with $\delta \ll \varepsilon$. Strictly speaking, one then gets a 'generalized' Milnor fibration for each germ $f_{t}$, which has the same homotopy type as the usual one and determines the embedded topology of the hypersurface germ defined by $f_{t}$ (see Proposition 3.7). We actually compare such generalized Milnor fibrations.

Now let us state our main results (proofs and complete statements can be found in $\S 4)$. We have chosen to treat the deformations $f_{t}=f+t g$ globally with respect to the parameter $t$, that is, $t \in \mathbb{P}^{1}=\mathbb{C} \cup\{\infty\}$ (with $f_{\infty}=g$ ). This can be justified by the natural appearance of these deformations in the study of the topology at infinity of a polynomial function $F \in \mathbb{C}[\mathbf{z}]$ (here, $t$ stands for the different values $F$ takes; see the Remark in paragraph 5.1). A more general motivation is that linear deformations can be understood as the local equations near a base point of divisors in a linear system defined on a smooth complex variety. We are then led to the following definition (see [15]).

Definition 1.1. Let $\Phi=(f, g):\left(\mathbb{C}^{n+1}, \mathbf{0}\right) \rightarrow\left(\mathbb{C}^{2}, \mathbf{0}\right)$ be a pair of germs of holomorphic functions without common factor. The pencil of hypersurface singularities $\mathscr{P}(\Phi)$ associated with $\Phi$ is the set of holomorphic germs of functions parametrized by the projective line $\mathbb{P}^{1}$ and defined by

$$
\mathscr{P}(\Phi):=\left\{\boldsymbol{\varphi}_{\tau}=\alpha g-\beta f \mid \tau=(\alpha: \beta) \in \mathbb{P}^{1}\right\} .
$$

The choice of a pair of homogeneous coordinates $(\alpha: \beta)$ for a parameter $\tau \in \mathbb{P}^{1}$ does not affect the local topology of the germ $\varphi_{\tau}$ (nor its analytic type): it is therefore well defined. We call the germs $\varphi_{\tau}$ the members of the pencil $\mathscr{P}(\Phi)$.

One can show, using the general results of Whitney and Thom-Mather on analytic stratifications (see for instance [14, 1.2.7 and 1.2.8]), that there is a finite subset $A_{\Phi}$ of the projective line $\mathbb{P}^{1}$ such that the embedded topology of the 
hypersurface germ $V\left(\varphi_{\tau}\right)$ defined by any member $\varphi_{\tau}$ with $\tau \in A_{\Phi}$ is different from those of all the $V\left(\varphi_{\tau^{\prime}}\right)$ with $\tau^{\prime} \notin A_{\Phi}$, which are all the same. A member $\varphi_{\tau}$ with $\tau \in A_{\Phi}$ is called atypical, and if $\tau \notin A_{\Phi}$ it is said to be generic. The study of the topology of a pencil then reduces to the following two questions.

(i) What is the finite set $A_{\Phi}$ of atypical parameters?

(ii) Is it possible to compare the atypical topologies with the generic ones?

When $\Phi=(f, g)$ is a pair with no blowing up, we obtain the following two results, related to these two questions. In this case, the pencil $\mathscr{P}(\Phi)$ is also said 'to have no blowing up'. This condition ensures that the discriminant Disc $\Phi$ is either void or a germ of a curve in $\left(\mathbb{C}^{2}, \mathbf{0}\right)$ (see the Remark following Example 3.3).

Definition 1.2. A member $\varphi_{\tau}$ of $\mathscr{P}(\Phi)$ is called transverse if $\tau$ does not belong to the projective tangent cone $\mathbb{P} C_{0}$ Disc $\Phi \subset \mathbb{P}^{1}$.

THEOREM 1.3. All transverse members of a pencil with no blowing up have isotopic Milnor fibrations. In particular, they are all generic. One then has the inclusion:

$$
A_{\Phi} \subset \mathbb{P} C_{\mathbf{0}} \operatorname{Disc} \Phi
$$

EXAMPLe 1.4. If $\Phi=(f, \ell)$, where $\ell$ is a generic linear form with respect to $f$, a lemma of Lê (see [9]) asserts that

$$
\mathbb{P} C_{\mathbf{0}} \operatorname{Disc}(f, \ell)=\{(0: 1)\} \text {. }
$$

But for $\tau=(1: t) \neq(0: 1), \varphi_{\tau}=\ell-t f$ is smooth. This gives therefore a special case of the preceding result.

Now for any member $\varphi_{\tau}$ of $\mathscr{P}(\Phi)$ we call any subfibration of its Milnor fibration with the same dimension and the same basis a Milnor subfibration of $\varphi_{\tau}$.

THEOREM 1.5. Every non-transverse member of a pencil with no blowing up has a Milnor subfibration (its internal Milnor subfibration, see Definition 4.1) isotopic to a Milnor subfibration of every transverse member.

The embedding theorems of $[\mathbf{2 5}, \mathbf{2 4}, \mathbf{2 7}]$ quoted above are special cases of this result. Note also that in the two main families of examples of pencils with no blowing up (pairs defining an isolated complete intersection singularity or giving generalized Iomdine-Lê deformations, see paragraph 5.1), the Milnor fibre is obtained from its internal Milnor subfibre by the addition of $n$-handles.

Theorem 1.5 makes it possible to describe a common factor between the characteristic polynomials of the top-dimensional homological monodromies of $f$ and $f_{t}=f+t g$, or more generally between their homological zeta functions (see Proposition 4.3). This was also noticed in $[\mathbf{2 5}, \mathbf{2 4}, \mathbf{2 2}, \mathbf{2 7}]$ in particular cases. Furthermore, if the pencil $\mathscr{P}(\Phi)$ has at least two non-transverse members, there is a Mayer-Vietoris spectral sequence which gives a theoretical way to compute the homological monodromy of the transverse member from those of the nontransverse members (see Theorem 4.5). This is the main result justifying the decision to consider the deformation parameter as an element of $\mathbb{P}^{1}$. 
Finally, in certain cases the Milnor fibre of one of the two compared members can be embedded in the Milnor fibre of the other (see Theorem 4.6 for a precise statement). This can lead to a finer approximation of the set of atypical members of $\mathscr{P}(\Phi)$ (see Corollary 4.7).

To conclude, we give in $\S 5$ two examples. The first describes generalised Iomdine-Lê deformations, with which we do not require $N$ to be large. The second is the study of a pair which does not fit in any of the families studied in the papers cited above.

Acknowledgements. This is a short version of my $\mathrm{PhD}$ thesis [3], a summary of which has already been published in [4]. I thank my advisor, Françoise Michel, for many useful discussions. I also thank Claude Weber and the Swiss National Fund, which provided financial support during the revision of a first version of this paper.

\section{Maximal isotopies of complex lines transverse to a plane curve germ}

Let $\Delta \subset\left(\mathbb{C}^{2}, \mathbf{0}\right)$ be a reduced plane curve germ $(\Delta=\operatorname{Disc}(f, g)$ in our applications). We will consider representatives of this germ, which we will still denote by $\Delta$, in a sufficiently small open ball $B_{\delta}=B_{\delta}^{4}$ with centre $\mathbf{0}$ and radius $\delta$. We can suppose that the only singular point of $\Delta$ is the origin $\mathbf{0}$. The purpose of this section is to describe isotopies of complex lines transverse to $\Delta$ in $B_{\delta}$.

The pair consisting of a complex line not containing $\mathbf{0}$ and of its intersection with $\Delta$ in $B_{\delta}$ is diffeomorphic to a (real) disc with a finite number of marked points on it. Such a pair will be called a marked disc. The notions of marked subdisc, of the marking of a marked (sub)disc and of isotopy of marked (sub) discs are defined in an obvious way.

A marked disc has a direction in the projective line $\mathbb{P}^{1}$ and a distance to the origin in $(0,+\infty)$. The internal marking of a marked (sub) disc $D$ of direction $\tau$ consists of the intersection of $D$ with the irreducible components of $\Delta$ whose tangent direction at $\mathbf{0}$ is different from $\tau$. This terminology comes from the fact that the intersection of $D$ with the components of $\Delta$ of direction $\tau$ at $\mathbf{0}$ is contained in an external corona of $D$ (see Figure 1).

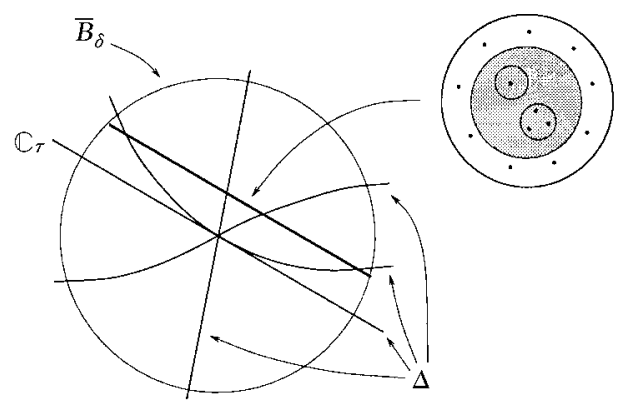

FIGURE 1. The internal marking (in the gray subdisc).

\subsection{Isotopies of families of marked subdiscs}

We will first consider isotopies between families of marked subdiscs. Here is the main result. 
Proposition 2.1. Let $\tau$ and $\tau^{\prime}$ be two directions in $\mathbb{P}^{1}$. In every sufficiently small open ball with centre $\mathbf{0}$ in $\mathbb{C}^{2}$, all marked discs with directions $\tau$ or $\tau^{\prime}$ which are sufficiently close to $\mathbf{0}$ have isotopic marked subdiscs, whose marking consists in the intersection of these discs with the components of $\Delta$ not tangent at 0 to $\tau$ or $\tau^{\prime}$.

In particular, if $\tau$ and $\tau^{\prime}$ do not belong to the projective tangent cone $\mathbb{P} C_{0} \Delta$ of $\Delta$ at $\mathbf{0}$, all these marked discs are isotopic.

Definition 2.2. A marked disc will be called transverse if its direction does not belong to the projective tangent cone $\mathbb{P} C_{\mathbf{0}} \Delta$.

The last proposition then says that all transverse marked discs sufficiently close to the origin are isotopic. Its proof comes from the following lemma.

Lemma 2.3. Let $\bar{\Omega} \subset \mathbb{P}^{1}$ be a smooth compact two-dimensional submanifold which meets the projective tangent cone $\mathbb{P}_{\mathbf{0}} \Delta$ of $\Delta$ at $\mathbf{0}$ in its interior. We denote this intersection by $\mathbb{P} C_{\mathbf{0}} \Delta_{\Omega}$. Then for all $\delta>0$ sufficiently small, the pair $\left(\bar{N}_{\bar{\Omega}, \delta}, \bar{N}_{\bar{\Omega}, \delta} \cap \Delta\right)$ is a pair of smooth manifolds with corners, where $\bar{N}_{\bar{\Omega}, \delta}:=\bar{B}_{\delta} \cap \mathbb{C} \bar{\Omega} \backslash\{\boldsymbol{0}\}$ is the cone over $\bar{\Omega}$ in the closed ball $\bar{B}_{\delta}$ with the origin removed.

Furthermore, let $K \subset \mathbb{P}^{1}$ be a compact subset which does not meet $\mathbb{P} C_{0} \Delta_{\Omega}$ nor the boundary $\partial \bar{\Omega}$. Then for all such $\delta>0$, there is an $r>0$ with $0<r \ll \delta$, such that every complex line of direction $\tau$ in $K$ whose distance to the origin lies in $(0, r)$ cuts this pair transversally.

Sketch of proof (see [3] for details). This is a consequence of the fact that, for every neighbourhood $V$ of $\mathbb{P} C_{0} \Delta_{\Omega}$ in $\Omega=\operatorname{Int}(\bar{\Omega})$, there is a $\delta>0$ such that the intersection $\bar{N}_{\bar{\Omega}, \delta} \cap \Delta$ is contained in the cone over $V$ in $\mathbb{C}^{2}$ (this property is true for all local affine complex varieties, regardless of their dimension) and every tangent line to this intersection has its direction in $V$ (this comes from the fact that the set of limiting tangent directions to $\Delta$ at $\mathbf{0}$ coincides precisely with the projective tangent cone; this is true only for curves). All the directions to be avoided in order for a line to cross the pair $\left(\bar{N}_{\bar{\Omega}, \delta}, \bar{N}_{\bar{\Omega}, \delta} \cap \Delta\right)$ transversally are then contained in $V \cup \partial \Omega$. Moreover, it follows from an elementary computation that for each $\delta>0$, there is an $r>0$ such that the intersection of $\bar{N}_{\bar{\Omega}}, \delta$ with a complex line with direction in $K$ and distance to the origin in $(0, r)$ is contained in the open ball $B_{\delta}$. One concludes by choosing $V$ disjoint from $K$.

Proof of Proposition 2.1. Let $K$ be a (smooth) path linking $\tau$ and $\tau^{\prime}$ in $\mathbb{P}^{1}$, and let $\bar{\Omega}$ be the complement in $\mathbb{P}^{1}$ of any sufficiently small contractible open neighbourhood of $K$ with smooth boundary, or the whole projective line $\mathbb{P}^{1}$, if neither $\tau$ nor $\tau^{\prime}$ lies in $\mathbb{P} C_{0} \Delta$. Then $\bar{\Omega}$ and $K$ fulfill the hypotheses of the preceding lemma. But its conclusion can be rephrased as follows: the set $\mathscr{B}_{K, r}$ of complex lines with direction in $K$ and distance to the origin in $(0, r)$ is a connected submanifold of the grassmannian $G_{\text {aff }}(1,2)$ of affine complex lines in $\mathbb{C}^{2}$, and the natural projection

$$
\pi_{\delta}: \bar{B}_{\delta} \times \mathbb{P}^{1} \rightarrow G_{\text {aff }}(1,2)
$$


sending a pair $(z, \tau)$ to the line passing through $z$ of direction $\tau$, when restricted to the pair $\left(\bar{N}_{\Omega, \delta}, \bar{N}_{\Omega, \delta} \cap \Delta\right) \times \mathbb{P}^{1}$, defines a proper submersion

$$
\pi_{\delta, K, r, \Omega}:\left(\left(\bar{N}_{\Omega, \delta}, \bar{N}_{\Omega, \delta} \cap \Delta\right) \times \mathbb{P}^{1}\right) \cap \pi^{-1}\left(\mathscr{B}_{K, r}\right) \rightarrow \mathscr{B}_{K, r}
$$

of pairs of smooth manifolds with corners, and thus a smooth fibration according to the Ehresmann fibration theorem. The proposition follows.

\subsection{Maximal isotopies of marked subdiscs}

Proposition 2.1 says in particular that two marked discs with arbitrary directions which are sufficiently close to the origin have a piece in common. We now determine a larger common piece. The point is that contrary to Proposition 2.1, this piece cannot be shared a priori by all the marked discs having one of the two directions and sufficiently close to $\mathbf{0}$. We can restrict ourselves to the comparison of a non-transverse marked disc with a transverse one.

Proposition 2.4. Let $\tau$ and $\tau^{\prime}$ be two distinct directions in $\mathbb{P}^{1}$, where $\tau$ is in the projective tangent cone $\mathbb{P} C_{\mathbf{0}} \Delta$ and $\tau^{\prime}$ is not. In any neighbourhood of $\mathbf{0}$ in $\mathbb{C}^{2}$ and for all $r>0$ sufficiently small, there are two marked discs $D$ and $D^{\prime}$ of directions $\tau$ and $\tau^{\prime}$ respectively and distance to the origin less than $r$, and an isotopy between a marked subdisc of $D$ containing its internal marking and a marked subdisc of $D^{\prime}$ containing all its marking except at most one point. This point corresponds to the case when the line going through $\mathbf{0}$ of direction $\tau$ is a component of $\Delta$. Furthermore, this isotopy can be made compatible with the one of Proposition 2.1.

Proof. This is an extended version of the 'rabattement dans le diagramme de Cerf' ('tilting up in the Cerf diagram') that appeared in [12].

First of all, after a linear change of coordinates, one can suppose that $\tau$ is the horizontal direction $(1: 0)$, that $\tau^{\prime}=(1: 1)$ and that the projective tangent cone $\mathbb{P} C_{0} \Delta$ does not contain the vertical direction $(0: 1)$. One can further assume that $\mathbb{P} C_{0} \Delta \subset\{(1: t)|| t \mid>1\}$, because of the isotopy of all the transverse discs. We will restrict ourselves to isotopies of the form $\left\{L_{t}^{y}\right\}_{t \in[0,1]}$, where $L_{t}^{y}$ is the complex line of direction $(1: t)$, and with $y \in \mathbb{C}$ as its intercept (in other words, the equation of $L_{t}^{y}$ is $\left.v=y+t u\right)$. The disc $D$ corresponding to $L_{0}^{y}$ will be called horizontal, while $D^{\prime}$, corresponding to $L_{1}^{y}$, will be called oblique. This may explain the name 'tilting up'.

We see that the set $\breve{\mathbb{C}}^{2}$ of non-vertical complex lines in $\mathbb{C}^{2}$ is isomorphic to $\mathbb{C}^{2}$, via the correspondence $(t, y) \mapsto L_{t}^{y}$. The distance to the origin of $L_{t}^{y}$ being smaller than $|y|$, the first step of the proof is to find a $y$ in an arbitrary small disc $D_{r}^{*} \subset \mathbb{C}$ such that all the complex lines $L_{t}^{y}$ with $t \in[0,1]$ meet $\Delta$ transversally. This is the same as asking that the segment $[0,1] \times\{y\}$ does not meet the dual variety $\check{\Delta} \subset \check{\mathbb{C}}^{2} \simeq \mathbb{C}^{2}$. The hypotheses made show that only the dual varieties $\check{\Delta}_{i}$ corresponding to the components $\Delta_{i}$ of $\Delta$ having horizontal directions at $\mathbf{0}$ can raise problems. But one can show (see [3, lemme II.12]) that the $\breve{\Delta}_{i}$ are complex curves passing through $\check{\mathbf{0}}$ with a horizontal direction at $\check{\mathbf{0}}$ in $\check{\mathbb{C}}^{2} \simeq \mathbb{C}^{2}$. A transversality argument can then be used to conclude this part of the proof.

The choice of $y$ being made, it remains to understand the common marking between the horizontal disc $D=L_{0}^{y} \cap B_{\delta}$ and the oblique disc $D^{\prime}=L_{1}^{y} \cap B_{\delta}$. It is 
indeed the case that the horizontal disc meets the components of $\Delta$ tangent to the $u$-axis (and distinct from it) in more points than the oblique disc does. On the contrary, the oblique disc meets the $u$-axis while the horizontal disc does not. An elementary but tedious study (see [3, II.3.3-3.4]) then shows that the common marking consists in all the marking of the oblique disc, except its intersection with the $u$-axis, when it is a component of $\Delta$, and that it contains the internal marking of the horizontal disc.

REMARK. A more detailed description of the common subdisc, corresponding to Figure 2, can be found in [3, II.4]. Note that this description was used implicitly in [26].
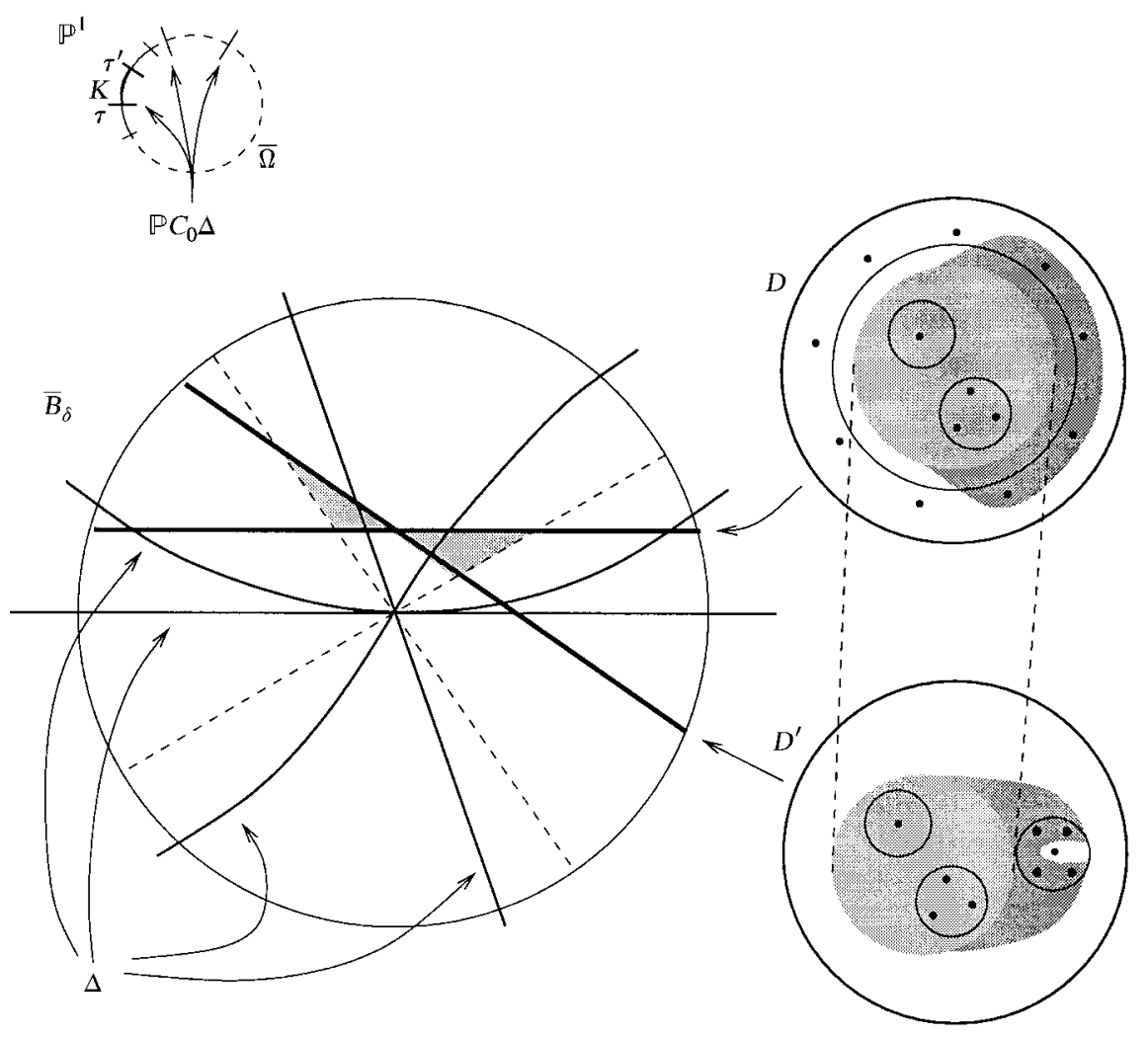

Figure 2. Marked discs 'tilting up'.

\section{Lifting isotopies within a pair with no blowing up}

The aim of this section is to give a sufficient condition on an analytic germ $\Phi=(f, g):\left(\mathbb{C}^{n+1}, \mathbf{0}\right) \rightarrow\left(\mathbb{C}^{2}, \mathbf{0}\right)$ in order to construct nice representatives of it, which means that when restricting to these, isotopies in $\left(\mathbb{C}^{2}, \mathbf{0}\right)$ can be lifted in $\left(\mathbb{C}^{n+1}, \mathbf{0}\right)$ under certain conditions. We will then use these representatives in the next section to deduce isotopies between the Milnor fibrations of two members of the pencil associated with $\Phi$ from the isotopies in $\left(\mathbb{C}^{2}, \mathbf{0}\right)$ constructed in the previous section. 


\subsection{Morphisms with no blowing up in codimension 0}

Recall first (see [7]) that the relative conormal space $C_{\Psi}(X)$ of a complex analytic mapping $\Psi:(X, \mathbf{0}) \rightarrow\left(\mathbb{C}^{p+1}, \mathbf{0}\right)$, defined in an open neighbourhood $X$ of $\mathbf{0}$ in $\mathbb{C}^{n+1}$, is the closure in the product $X \times \check{\mathbb{P}}^{n}$ of the set of hyperplanes tangent to the smooth parts of the fibres of $\Psi$. Such a hyperplane is given by a point $z \in X$ of tangency with a fibre of $\Psi$ and its direction $\check{\tau} \in \check{\mathbb{P}}^{n}$ in the projective space of hyperplane directions in $\mathbb{C}^{n+1}$. This set $C_{\Psi}(X)$ is analytic in $X \times \check{\mathbb{P}}^{n}$ and is endowed with a natural projection $\tau_{\Psi}: C_{\Psi}(X) \rightarrow X$. The fibre of $\tau_{\Psi}$ over $z \in X$ is exactly the set of limiting tangent hyperplane directions in $\breve{\mathbb{P}}^{n}$ at $z$ to the fibres of $\Psi$.

Definition 3.1 [7]. A complex analytic germ $\Psi:\left(\mathbb{C}^{n+1}, \mathbf{0}\right) \rightarrow\left(\mathbb{C}^{p+1}, \mathbf{0}\right)$ has no blowing up (in codimension 0 ) if it is open and if the composition $\Psi \circ \tau_{\Psi}: C_{\Psi}(X) \rightarrow \mathbb{C}^{p+1}$ of a representative of $\Psi$ with the natural projection of its relative conormal space is open.

Lemma 3.2. A complex analytic germ $\Psi:\left(\mathbb{C}^{n+1}, \mathbf{0}\right) \rightarrow\left(\mathbb{C}^{p+1}, \mathbf{0}\right)$ has no blowing up if and only if its zero locus $V(\Psi)$ admits a (complex analytic) Whitney stratification along which the non-critical locus $\mathbb{C}^{n+1} \backslash$ Crit $\Psi$ satisfies Thom's

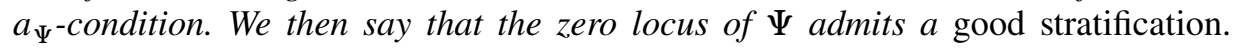

Proof. By hypothesis, $\Psi$ is open, and thus equidimensional, when it has no blowing up. One can then apply Theorem 7.1.1 of [7] to show that its zero locus admits a good stratification.

Now if $V(\Psi)$ admits a good stratification $\mathscr{S}$, any limit at a point $z$ of $V(\Psi)$ of hyperplanes tangent to the fibres of $\Psi$ is tangent to the stratum $S$ containing it. In other words, the fibre at $z$ of the relative conormal space is contained in the fibre of the ('absolute') conormal space of $S$ (that is, the set of hyperplanes containing the tangent space to $S$ at $z$ ). Thus, the union of the fibres of the relative conormal space at the points of $V(\Psi)$, that is, the fibre at $\mathbf{0}$ of the composition $\Psi \circ \tau_{\Psi}$, is contained in the finite union of the conormal spaces of the strata of $\mathscr{S}$, which are known to be $n$-dimensional. Since any fibre of $\Psi \circ \tau_{\Psi}$ at a regular value of $\Psi$ is $n$-dimensional (as the conormal space to the fibre of $\Psi$ at this point), and since the dimension of the fibres of $\Psi \circ \tau_{\Psi}$ is a lower semi-continuous function, this composition is equidimensional, and thus open, over any sufficiently small neighbourhood of $\mathbf{0}$ in $\mathbb{C}^{p+1}$.

Examples 3.3. (a) $(p=0)$ Any germ $f:\left(\mathbb{C}^{n+1}, \mathbf{0}\right) \rightarrow(\mathbb{C}, \mathbf{0})$ of a function has no blowing up (any Whitney stratification of the zero locus $V(f)$ is in fact a good stratification; see [2]).

(b) Any germ $\Psi$ defining an isolated complete intersection singularity has no blowing up (this can be seen by applying the preceding characterization). In particular, this condition is always fulfilled when $\Psi$ is finite (that is, $p=n$ and $\Psi$ is open).

(c) If the germ $\Psi:\left(\mathbb{C}^{n+1}, \mathbf{0}\right) \rightarrow\left(\mathbb{C}^{p+1}, \mathbf{0}\right)$ has no blowing up, then for a generic linear projection $\ell:\left(\mathbb{C}^{n+1}, \mathbf{0}\right) \rightarrow\left(\mathbb{C}^{q}, \mathbf{0}\right)$, the germ $(\Psi, \ell):\left(\mathbb{C}^{n+1}, \mathbf{0}\right) \rightarrow$ $\left(\mathbb{C}^{p+q+1}, \mathbf{0}\right)$ has no blowing up (this is a basic tool in the study of the polar varieties of Lê and Teissier; see for instance [14]). 
(d) The composition of a finite germ $\Pi:\left(\mathbb{C}^{p+1}, \mathbf{0}\right) \rightarrow\left(\mathbb{C}^{p+1}, \mathbf{0}\right)$ with a germ $\Psi:\left(\mathbb{C}^{n+1}, \mathbf{0}\right) \rightarrow\left(\mathbb{C}^{p+1}, \mathbf{0}\right)$ with no blowing up again has no blowing up (apply the preceding lemma).

(e) Any direct product $\Psi_{1} \times \Psi_{2}:\left(\mathbb{C}^{n_{1}+n_{2}+2}, \mathbf{0}\right) \rightarrow\left(\mathbb{C}^{p_{1}+p_{2}+2}, \mathbf{0}\right)$ of two germs with no blowing up is again with no blowing up (the product of the good stratifications of $V\left(\Psi_{1}\right)$ and $V\left(\Psi_{2}\right)$ is a good stratification of $V\left(\Psi_{1} \times \Psi_{2}\right)$ ).

(f) (Lê) The germ $(x, y, z) \mapsto\left(x, y^{2}-x z\right)$ is open, homogeneous but with blowing up (consider the points $\left(t, t^{2}, c\right)$ tending towards a point $(0,0, c)$ in the zero locus and the limiting tangent direction associated with this sequence).

Remark. The discriminant of the last example is just the origin $\mathbf{0}$ in $\mathbb{C}^{2}$, while those of the first two examples are hypersurfaces in $\mathbb{C}^{p+1}$ (see for instance $[\mathbf{1 7}, 2.8]$ in the second case). This is indeed a general fact (see $[\mathbf{1 6}, 1.3 .2])$ : the discriminant of a germ $\Psi:\left(\mathbb{C}^{n+1}, \mathbf{0}\right) \rightarrow\left(\mathbb{C}^{p+1}, \mathbf{0}\right)$ with no blowing up is either void or a hypersurface germ in $\left(\mathbb{C}^{p+1}, \mathbf{0}\right)$.

Now let us state the main property of germs with no blowing up. Notice that for $p=0$ this is exactly the Milnor fibration theorem.

Proposition 3.4. Let $\Psi:\left(\mathbb{C}^{n+1}, \mathbf{0}\right) \rightarrow\left(\mathbb{C}^{p+1}, \mathbf{0}\right)$ be a complex analytic germ with no blowing up. For any $\varepsilon>0$ sufficiently small, there is a $\delta>0$, with $\delta \ll \varepsilon$, such that the restriction

$$
\Psi_{\varepsilon, \delta}: \bar{B}_{\varepsilon}^{2 n+2} \cap \Psi^{-1}\left(\bar{B}_{\delta}^{2 p+2} \backslash \operatorname{Disc} \Psi\right) \stackrel{\Psi}{\longrightarrow} \bar{B}_{\delta}^{2 p+2} \backslash \operatorname{Disc} \Psi
$$

is a locally trivial smooth fibration.

Proof. This is an easy consequence of the $a_{\Psi}$-condition satisfied by $\mathbb{C}^{n+1} \backslash$ Crit $\Psi$ along a good stratification of $V(\Psi)$, and the transversality of this stratification with any sufficiently small sphere with centre $\mathbf{0} \in \mathbb{C}^{n+1}$. Details are left to the reader.

3.2. An isotopy lemma for pairs $(f, g):\left(\mathbb{C}^{n+1}, \mathbf{0}\right) \rightarrow\left(\mathbb{C}^{2}, \mathbf{0}\right)$ with no blowing up

Proposition 3.4 is the first step towards the lifting of isotopies in $\left(\mathbb{C}^{p+1}, \mathbf{0}\right)$ to isotopies in $\left(\mathbb{C}^{n+1}, \mathbf{0}\right)$. Unfortunately, when dealing with isotopies of hypersurfaces, the intersections of these with the discriminant Disc $\Psi$ in $\mathbb{C}^{p+1}$ are not tractable without studying their intersection with the boundary sphere $S_{\delta}^{2 p+1} \subset \mathbb{C}^{p+1}$, unless $p \leqslant 1$. This is the first reason why we are primarily concerned with pairs $\Phi=(f, g)$ with no blowing up. The second will appear in the proof of the following proposition, which is the technical core of our work.

Proposition 3.5. Let $\Phi=(f, g):\left(\mathbb{C}^{n+1}, \mathbf{0}\right) \rightarrow\left(\mathbb{C}^{2}, \mathbf{0}\right)$ be a pair with no blowing up. Then for all $\varepsilon>0$ sufficiently small there is a $\delta>0$, with $\delta \ll \varepsilon$, such that the representative

$$
\Phi_{\varepsilon, \delta}: \bar{X}_{\varepsilon, \delta}:=\bar{B}_{\varepsilon}^{2 n+2} \cap \Phi^{-1}\left(\bar{B}_{\delta}^{4}\right) \rightarrow \bar{B}_{\delta}^{4}
$$

lifts the isotopies. By this we mean that, for any submanifold $Q$ of $\bar{B}_{\delta}^{4}$ meeting Disc $\Phi$ transversally outside of $\mathbf{0}$, one has the following: 
(1) the preimage $P:=\Phi_{\varepsilon, \delta}^{-1}(Q)$ is a submanifold of $\bar{X}_{\varepsilon, \delta}$;

(2) for any proper (stratified) submersion $\Pi:(Q, Q \cap \operatorname{Disc} \Phi) \rightarrow R$, the composition $\Pi \circ \Phi_{\varepsilon, \delta} \mid: P \rightarrow R$ is also a proper submersion.

Proof. To prove the requirement (1) (and by the way that $\bar{X}_{\varepsilon, \delta}$ is indeed a manifold with corners), it suffices to show that all the faces $Q_{i}$ composing the manifold with corners $Q$ are transverse to the restrictions of $\Phi_{\varepsilon, \delta}$ to the two faces of the manifold with boundary $\bar{B}_{\varepsilon}^{2 n+2}$, that is, $B_{\varepsilon}^{2 n+2}$ and $S_{\varepsilon}^{2 n+1}$. Since $\Phi$ has no blowing up, these two restrictions are submersive over the complement of Disc $\Phi$ in $B_{2 \delta}^{4}$, for $\delta$ sufficiently small. Then we just have to check what happens over the intersection of $Q$ with $\operatorname{Disc} \Phi$. So let $w \in Q \cap \operatorname{Disc} \Phi$ and $z \in \Phi_{\varepsilon, \delta}^{-1}(w)$. By hypothesis, $w$ is different from $\mathbf{0}$; hence it is a smooth point of Disc $\Phi$. One can then show, using the Curve Selection Lemma, that $\operatorname{im} d \Phi(z) \supseteq T_{w} \operatorname{Disc} \Phi$ (see [3, I.41]). But the face $Q_{i}$ of $Q$ containing $w$ meets Disc $\Phi$ transversally. So we have $T_{w} \operatorname{Disc} \Phi+T_{w} Q_{i}=T_{w} \bar{B}_{\delta}^{4}$, which gives

$$
\operatorname{im}(d \Phi(z))+T_{w} Q_{i}=T_{w} \bar{B}_{\delta}^{4}
$$

If $z \in B_{\varepsilon}^{2 n+2}$, then $\operatorname{im} d \Phi_{\varepsilon, \delta}(z)=\operatorname{im} d \Phi(z)$, and the result follows.

If $z \in S_{\varepsilon}^{2 n+1}$, it suffices to show that $\left.\operatorname{im} d \Phi\right|_{S_{\varepsilon}^{2 n+1}}(z)=\operatorname{im} d \Phi(z)$, in other words $\operatorname{ker} d \Phi(z)+T_{z} S_{\varepsilon}^{2 n+1}=T_{z} \mathbb{C}^{n+1}$. But $z \notin V(\Phi)$, so if $\delta$ and $\varepsilon$ are chosen such that $f_{\varepsilon, \delta}$ and $g_{\varepsilon, \delta}$ are Milnor fibrations for $f$ and $g$ (see the Introduction), $z$ is a regular point of $f$ or of $g$ (for instance of $f$ ), and the kernel $\operatorname{ker} d \Phi(z)$ is exactly the tangent space to the fibre of $f$ containing $z$. But this fibre is transverse to the sphere $S_{\varepsilon}^{2 n+1}$, which establishes the property. Note here the importance of $\Phi$ being a pair.

To prove the second requirement, we just have to show that the restriction of the composition $\Pi \circ \Phi_{\varepsilon, \delta}$ to all the faces of the manifold with corners $P$ is a submersion, the properness being clear. Let $z$ be a point in a face $P_{i, j}$ of $P$ over the face $Q_{i}$ of $Q$. One has the following chain of linear maps:

$$
T_{z} P_{i, j} \stackrel{\left.d \Phi_{\varepsilon, \delta}\right|_{P_{i, j}}}{\longrightarrow} T_{\Phi(z)} Q_{i} \stackrel{d \Pi(\Phi(z))}{\longrightarrow} T_{\Pi(\Phi(z))} R .
$$

Now the intersection

$$
T_{\Phi(z)} Q_{i} \cap T_{\Phi(z)} \operatorname{Disc} \Phi=T_{\Phi(z)}\left(Q_{i} \cap \operatorname{Disc} \Phi\right)
$$

is contained in the image $\operatorname{im}\left(\left.d \Phi_{\varepsilon, \delta}\right|_{P_{i, j}}(z)\right)$. Since $\Pi$ is a submersion of the pair $(Q, Q \cap \operatorname{Disc} \Phi)$ on $R$, this concludes the proof.

\subsection{Generalized Milnor fibrations for the members of a pencil with no blowing up}

For a fixed pair $\Phi=(f, g)$ with no blowing up, Proposition 3.5 gives us a fundamental family of privileged neighbourhoods of $\mathbf{0}$ in $\mathbb{C}^{n+1}$ which have very nice properties. It is then natural to ask to what extent we can restrict to these neighbourhoods when considering the Milnor fibrations of the different members of the pencil associated with $\Phi$. The following Lemma 3.8 says that it is possible to do so, provided we consider only a finite number of members.

We first need some technical preparations. The question of whether the Milnor fibration of a complex analytic germ of the function $f:\left(\mathbb{C}^{n+1}, \mathbf{0}\right) \rightarrow(\mathbb{C}, 0)$ is well defined has been carefully analysed by Massey in [20, II]. But we have to adapt 
his results to show that the 'new' Milnor fibration determines the embedded topological type of $V(f)$ in $\left(\mathbb{C}^{n+1}, \mathbf{0}\right)$. Durfee in [5] introduced the notion of algebraic neighbourhood and showed the invariance of the embedded topological type with respect to the choice of the function defining these neighbourhoods. To use this result, we have to introduce the notion of 1-parameter family of privileged subanalytic neighbourhoods, which was inspired by [14, 2.2]. So the Definition and the Proposition that follow are merely a rephrasing of the results of Lê and Teissier, Durfee, and Massey that we have just cited.

Definition 3.6. Let $X$ be an open neighbourhood of $\mathbf{0}$ in $\mathbb{C}^{n+1}$. A subanalytic rug function $\alpha: X \rightarrow \mathbb{R}_{+}$is a subanalytic, proper function such that $\alpha^{-1}(0)=\{\mathbf{0}\}$. A (closed) subanalytic neighbourhood of $\mathbf{0}$ in $\mathbb{C}^{n+1}$ is a compact neighbourhood $\bar{U}_{\varepsilon}(\alpha)$ of $\mathbf{0}$ in $\mathbb{C}^{n+1}$ of the form

$$
\bar{U}_{\varepsilon}(\alpha):=\alpha^{-1}([0, \varepsilon]),
$$

where $\alpha$ is a subanalytic rug function and $\varepsilon>0$ a sufficiently small positive real number.

Now let $f:\left(\mathbb{C}^{n+1}, \boldsymbol{0}\right) \rightarrow(\mathbb{C}, 0)$ be a germ of an analytic function. A subanalytic neighbourhood $\bar{U}_{\varepsilon}(\alpha)$ of $\mathbf{0}$ in $\mathbb{C}^{n+1}$ is called privileged with respect to $f$ if it admits a Whitney stratification such that its interior $U_{\varepsilon}(\alpha)$ is a stratum of this stratification, and whose strata meet the strata of a Whitney stratification of $V(f)$ transversally. Finally, we say that the subanalytic rug function $\alpha: X \rightarrow \mathbb{R}_{+}$defines a 1-parameter family of privileged neighbourhoods with respect to $f$ if its graph $\operatorname{gr}(\alpha) \subset X \times \mathbb{R}_{+}$admits a subanalytic stratification the restriction of which makes each $\bar{U}_{\varepsilon}(\alpha)$ a privileged neighbourhood with respect to $f$, for all $\varepsilon>0$ sufficiently small.

Proposition 3.7. Let $f:\left(\mathbb{C}^{n+1}, \mathbf{0}\right) \rightarrow(\mathbb{C}, 0)$ be a germ of an analytic function. Let $\alpha: X \rightarrow \mathbb{R}_{+}$be a subanalytic function defining a 1-parameter family of privileged neighbourhoods with respect to $f$. Then for all $\varepsilon>0$ sufficiently small, there is $\eta>0$, with $\eta \ll \varepsilon$, such that the restriction

$$
f_{\varepsilon, \eta}: \bar{U}_{\varepsilon}(\alpha) \cap f^{-1}\left(\bar{D}_{\eta}^{*}\right) \stackrel{f}{\longrightarrow} \bar{D}_{\eta}^{*}
$$

is a locally trivial topological (stratified) fibration, whose fibre homotopy type does not depend on the choice of $\alpha$. Furthermore, its total space gives the embedded topological type of $V(f)$ in $\left(\mathbb{C}^{n+1}, \mathbf{0}\right)$. We will call such a restriction the (generalized) Milnor fibration of $f$.

Proof. The first part of the statement is exactly Proposition 1.5 of [20], in the light of the result of Briançon, Maisonobe and Merle quoted above that any Whitney stratification of $V(f)$ satisfies the $a_{f}$ condition.

Using the terminology of [6], we see that this part of the statement shows that the set of pairs $(\varepsilon, \eta) \in \mathbb{R}_{+}^{2}$ such that $f_{\varepsilon, \eta}$ is a stratified submersion is fringed. But because we are considering a 1-parameter family of privileged neighbourhoods, this set can be shown to be subanalytic (see [3, proposition [I.21]). It therefore contains the graph of a strictly increasing subanalytic function $\eta_{f, \alpha}$. Now, to show that the fibration $f_{\varepsilon, \eta_{f, \alpha}}$ determines the topological type of $V(f)$ in $\left(\mathbb{C}^{n+1}, \mathbf{0}\right)$, it suffices to notice that its total space is the complement of $V(f)$ in the subanalytic 
neighbourhood $\bar{U}_{\varepsilon}(\beta)$ associated with the subanalytic function

$$
\beta:=\max \left(\alpha, \eta_{f, \alpha}^{-1}(|f|)\right) \text {. }
$$

We then conclude by using the fundamental result of Durfee [5, 3.5], which extends naturally to the subanalytic context.

Proposition 3.7, and the (simple) fact that the ordinary balls $\bar{B}_{\varepsilon}^{2 n+2}$ form a 1parameter family of privileged neighbourhoods with respect to any germ $f$, show that the study of the Milnor fibration can be done in any 1-parameter family of privileged neighbourhoods. Now we come to the result we were aiming at.

Lemma 3.8. Let $\tau \subset \mathbb{P}^{1}$ be a finite subset of the projective line. For any germ $\Phi:\left(\mathbb{C}^{n+1}, \mathbf{0}\right) \rightarrow\left(\mathbb{C}^{2}, \mathbf{0}\right)$ with no blowing up, there are a number $\varepsilon_{\Phi}>0$ and a strictly increasing subanalytic function $\delta_{\Phi}:\left[0, \varepsilon_{\Phi}\right] \rightarrow \mathbb{R}_{+}$such that, for all $\varepsilon \in\left(0, \varepsilon_{\Phi}\right]$, the neighbourhoods $\bar{X}_{\varepsilon}:=\bar{B}_{\varepsilon}^{2 n+2} \cap \Phi^{-1}\left(\bar{B}_{\delta_{\Phi}(\varepsilon)}^{4}\right)$ satisfy the requirements of Proposition 3.5 and form a 1-parameter family of privileged neighbourhoods with respect to all the members $\varphi_{\tau}$ of $\mathscr{P}(\Phi)$ with $\tau \in \tau$.

Proof. Let us first examine the stratification of the neighbourhoods $\bar{X}_{\varepsilon, \delta}$ of Proposition 3.5. They are manifolds with corners, and their natural stratification is

$$
\begin{aligned}
\bar{X}_{\varepsilon, \delta}= & \left(B_{\varepsilon}^{2 n+2} \cap \Phi^{-1}\left(B_{\delta}^{4}\right)\right) \cup\left(B_{\varepsilon}^{2 n+2} \cap \Phi^{-1}\left(S_{\delta}^{3}\right)\right) \\
& \cup\left(S_{\varepsilon}^{2 n+1} \cap \Phi^{-1}\left(B_{\delta}^{4}\right)\right) \cup\left(S_{\varepsilon}^{2 n+1} \cap \Phi^{-1}\left(S_{\delta}^{3}\right)\right) .
\end{aligned}
$$

Moreover, if $\delta$ is given by a subanalytic function $\delta_{\Phi}$, the graph of the subanalytic function $\alpha=\max \left(r, \delta_{\Phi}^{-1}(|\Phi|)\right)$ defining these neighbourhoods is also a manifold with corners, whose strata correspond exactly to the preceding decomposition. By the same 'fringed' argument as in the proof of Proposition 3.7, and by the finiteness of $\tau$, we just have to check that for all $\varepsilon>0$ sufficiently small and $\delta \ll \varepsilon$ there is a Whitney stratification of $V\left(\varphi_{\tau}\right)$ meeting all the strata of $\bar{X}_{\varepsilon, \delta}$ transversally for a fixed $\tau \in \tau$.

Note that every complex analytic Whitney refinement of a good stratification of $V(\Phi)$ is again good. Let $\widetilde{\tau}$ be a non-zero element of the orthogonal space $\tau^{\perp \mathbb{C}}$ in $\mathbb{C}^{2}$. We now fix a Whitney stratification $\boldsymbol{\Sigma}$ of $V\left(\varphi_{\tau}\right) \cup V\left(\varphi_{\tilde{\tau}}\right)$ such that $V(\Phi)$ is a union of strata refining its original good stratification. Let $\varepsilon>0$ and $\delta \ll \varepsilon$ be sufficiently small for $S_{\varepsilon}^{2 n+1}$ to be transverse to all the strata of this stratification and for $S_{\delta}^{3}$ to be transverse to all the branches of Disc $\Phi$. Let $\Sigma_{\varphi_{\tau}}$ be a stratum of $V\left(\varphi_{\tau}\right)$, and let $z \in \Sigma_{\varphi_{\tau}}$.

If $z \in\left(S_{\varepsilon}^{2 n+1} \cap \Phi^{-1}\left(B_{\delta}^{4}\right)\right)$, the transversality of the sphere $S_{\varepsilon}^{2 n+1}$ with all the strata of the Whitney stratification $\Sigma$ of $V\left(\varphi_{\tau}\right) \cup V\left(\varphi_{\tilde{\tau}}\right)$ suffices to conclude the proof.

If $z \in\left(B_{\varepsilon}^{2 n+2} \cap \Phi^{-1}\left(S_{\delta}^{3}\right)\right)$, then $z \notin V(\Phi)$ and so, for $\varepsilon>0$ sufficiently small, the (complex) rank of the restriction $\left.\Phi\right|_{\Sigma_{\varphi_{\tau}}}$ is 1 (this is a simple application of the Curve Selection Lemma). The transversality of Disc $\Phi$ with the sphere $S_{\delta}^{3}$ then implies the transversality of $\Sigma_{\varphi_{\tau}}$ with $\left(B_{\varepsilon}^{2 n+2} \cap \Phi^{-1}\left(S_{\delta}^{3}\right)\right)$.

If $z \in\left(S_{\varepsilon}^{2 n+1} \cap \Phi^{-1}\left(S_{\delta}^{3}\right)\right)$, we must show the transversality

$$
T_{z} \Sigma_{\varphi_{\tau}} \pitchfork\left(T_{z} \Phi^{-1}\left(S_{\delta}^{3}\right) \cap T_{z} S_{\varepsilon}^{2 n+1}\right) .
$$


These three spaces being pairwise transverse, it is equivalent to show that

$$
\left(T_{z} \Sigma_{\varphi_{\tau}} \cap T_{z} \Phi^{-1}\left(S_{\delta}^{3}\right)\right) \pitchfork T_{z} S_{\varepsilon}^{2 n+1} .
$$

But, because $\Phi(z) \in \mathbb{C} \tau$, one has $T_{\Phi(z)} S_{\delta}^{3} \supset \mathbb{C} \widetilde{\tau}$, which, by taking the inverse images, implies the inclusion $T_{z} \Phi^{-1}\left(S_{\delta}^{3}\right) \supset T_{z} \varphi_{\tilde{\tau}}^{-1}\left(\varphi_{\widetilde{\tau}}(z)\right)$. Thus, it suffices to show that, for $\Phi(z)$ sufficiently small, one has

$$
\left(T_{z} \Sigma_{\varphi_{\tau}} \cap T_{z} f_{\widetilde{\tau}}^{-1}\left(\varphi_{\widetilde{\tau}}(z)\right)\right) \pitchfork T_{z} S_{\varepsilon}^{2 n+1} .
$$

So let $z_{\infty}$ be in $V(\Phi) \cap S_{\varepsilon}^{2 n+1}$. Let $\Sigma$ be the stratum of $\Sigma$ containing it. Suppose that there is a sequence $\left(z_{k}\right)$ tending to $z_{\infty}$ such that:

(i) there is a stratum $\Sigma_{\varphi_{\tau}}$ of $V\left(\varphi_{\tau}\right)$ such that $z_{k} \in S_{\varepsilon}^{2 n+1} \cap \Sigma_{\varphi_{\tau}}$ for all $k$,

(ii) the sequence of tangent spaces $\left(T_{z_{k}} \Sigma_{\varphi_{\tau}}\right)$ converges,

(iii) the sequence of tangent spaces $\left(T_{z_{k}} \varphi_{\tilde{\tau}}^{-1}\left(\varphi_{\tilde{\tau}}\left(z_{k}\right)\right)\right)$ to the fibres of $\varphi_{\tilde{\tau}}$ at the $z_{k}$ converges,

(iv) for all $k$, one has the non-transversality

$$
\left(T_{z_{k}} \Sigma_{\varphi_{\tau}} \cap T_{z_{k}} \varphi_{\tilde{\tau}}^{-1}\left(\varphi_{\tau}\left(z_{k}\right)\right)\right) \not T_{z_{k}} S_{\varepsilon}^{2 n+1} .
$$

Since the stratification $\Sigma$ is of Whitney type, one has

$$
\lim \left(T_{z_{k}} \Sigma_{\varphi_{\tau}}\right) \supset T_{z_{\infty}} \Sigma \text {. }
$$

Moreover, since the restriction of the stratification $\Sigma$ to $V\left(\varphi_{\tilde{\tau}}\right)$ is a $\operatorname{good}$ stratification (being of Whitney type), one has $\lim \left(T_{z_{k}} \varphi_{\widetilde{\tau}}^{-1}\left(\varphi_{\widetilde{\tau}}\left(z_{k}\right)\right)\right) \supset T_{z_{\infty}} \Sigma$. Because $T_{z_{\infty}} S_{\varepsilon}^{2 n+1} \not T_{z_{\infty}} \Sigma$, one gets a contradiction.

\section{Common Milnor subfibres and subfibrations between members of a pencil of hypersurface singularities}

Let $\Phi=(f, g):\left(\mathbb{C}^{n+1}, \mathbf{0}\right) \rightarrow\left(\mathbb{C}^{2}, \mathbf{0}\right)$ be a germ with no blowing up. Lemma 3.8, together with Propositions 3.7 and 3.5, allows one to deduce isotopies between the Milnor fibrations of a finite number of members $\varphi_{\tau}$ of the pencil $\mathscr{P}(\Phi)$ associated with $\Phi$ from the isotopies between complex lines in $\mathbb{C}^{2}$ constructed in $\S 2$. We will now prove the corresponding results, which were for the most part stated in the Introduction.

\subsection{Comparison between the Milnor fibrations of a non-transverse member and of the transverse one}

If we consider a non-transverse member, we cannot construct a priori an isotopy as in Theorem 1.3 between the associated Milnor fibration and that of a transverse member. Nevertheless, they will have a piece in common. First, let us fix some notation: if $\varphi_{\tau}$ is a non-transverse member, the total space of its Milnor fibration, defined in a suitable $\bar{X}_{\varepsilon}$ as in Lemma 3.8, will simply be denoted by $E_{\tau}$, and its fibre by $F_{\tau}$. Similarly, and because of Theorem $1.3, \varphi_{\text {trans }}, E_{\text {trans }}$ and $F_{\text {trans }}$ will refer to a transverse member, the total space of its Milnor fibration and its Milnor fibre, respectively.

Definition 4.1. Let $U_{\tau} \subset \mathbb{P}^{1}$ be an open contractible neighbourhood of $\tau$ with smooth boundary containing no other points of $\mathbb{P} C_{0} \operatorname{Disc} \Phi$. Let $E_{\tau}^{\text {int }}$ be the 
submanifold of the total space $E_{\tau}$ of the Milnor fibration of $\varphi_{\tau}$ given by

$$
E_{\tau}^{\text {int }}:=\left\{z \in E_{\tau} \mid(f(z): g(z)) \notin U_{\tau}\right\} .
$$

The restriction of $\varphi_{\tau}$ to $E_{\tau}^{\text {int }}$ defines a Milnor subfibration of $\varphi_{\tau}$ whose topological type does not depend on the choice of $U_{\tau} \subset \mathbb{P}^{1}$. We will call it the internal Milnor subfibration of the non-transverse member $\varphi_{\tau}$.

The fibre $F_{\tau}^{\text {int }}$ of this subfibration is called the internal Milnor subfibre of $\varphi_{\tau}$. Notice that the submanifold $E_{\tau}^{\mathrm{int}}$ contains the intersection of $E_{\tau}$ with all the components of the critical locus of $\Phi$, except those whose image by $\Phi$ have $\tau$ as tangent direction at $\mathbf{0}$.

EXAMPLE 4.2. If the only component of Disc $\Phi$ having $\tau$ as tangent direction at $\mathbf{0}$ is the complex line with direction $\tau$, the Milnor fibration of $\varphi_{\tau}$ is homeomorphic to its internal subfibration (this comes from the equality $\left.E_{\tau} \cap \operatorname{Crit} \Phi=E_{\tau}^{\text {int }} \cap \operatorname{Crit} \Phi\right)$.

Remark. To show that $E_{\tau}^{\text {int }}$ defines a Milnor subfibration of $\varphi_{\tau}$ which does not depend on the choice of $U_{\tau}$, or that $E_{\tau}^{\text {int }} \simeq E_{\tau}$ in the preceding example, one can use the isotopy Lemma 3.5; this may constitute for the reader a warm-up before the proof that comes next, which is based on the same principle and which is detailed.

Proofs of Theorems 1.3 and 1.5. Let us prove Theorem 1.5 first. We can suppose after a change of coordinates that the non-transverse member $\varphi_{\tau}$ is $g$, that the member $f+g$ is transverse, and that there are no non-transverse parameters other than $(1: 0)$ in the disc $\bar{U}_{g}:=\{(1: t)|| t \mid \leqslant 1+c\}$, with $c>0$ small. Let then $\bar{X}_{\varepsilon}$ be a privileged neighbourhood for both $g$ and $f+g$, as in Lemma 3.8. Consider the following unfolding of $g$ :

$$
G:\left(\begin{array}{rl}
\bar{X}_{\varepsilon} \times[0,1] & \rightarrow(\mathbb{C}, 0) \times[0,1] \\
(z, t) & \mapsto(t f(z)+g(z), t)
\end{array}\right) .
$$

A way to obtain two isotopic Milnor subfibrations for $g_{0}=g$ and $g_{1}=f+g$ is to construct a submanifold $P \subset \bar{X}_{\varepsilon} \times[0,1]$ fibred by the restriction of $G$. But $\Phi_{\varepsilon}$ factors the unfolding $G$ naturally: one has $G=H \circ\left(\Phi_{\varepsilon} \times \operatorname{Id}_{[0,1]}\right)$, with

$$
H:\left(\begin{array}{c}
\bar{B}_{\delta_{\Phi}(\varepsilon)}^{4} \times[0,1] \rightarrow(\mathbb{C}, 0) \times[0,1] \\
((u, v), t) \mapsto(t u+v, t)
\end{array}\right) .
$$

Moreover, $\Phi_{\varepsilon}$ lifts the isotopies according to Proposition 3.5; hence the product $\Phi_{\varepsilon} \times \operatorname{Id}_{[0,1]}$ does too. Therefore, it suffices to construct a submanifold $Q$ of $\bar{B}_{\delta_{\Phi}(\varepsilon)}^{4} \times[0,1]$ such that the restriction of $H$ to the pair $(Q, Q \cap(\operatorname{Disc} \Phi \times[0,1]))$ is a proper submersion.

But now the fibres of $H$ are exactly the pairs $H^{-1}(y, t)=\left(L_{t}^{y}, t\right)$, where $t \in[0,1]$ and $L_{t}^{y}$ is the complex line with slope $t$ and with origin ordinate $y$. We can then use Lemma 2.3 (with $\bar{\Omega}=\mathbb{P}^{1} \backslash U_{g}=\{(s: 1)|| s \mid \leqslant 1 /(1+c)\}$, and $K=[(1: 0),(1: 1)])$ to show that when $|y| \neq 0$ is sufficiently small, all these lines are transverse to the pair $(N, N \cap \operatorname{Disc} \Phi)$, where

$$
N:=\left\{(u, v) \in \bar{B}_{\delta_{\Phi}(\varepsilon)}^{4} \backslash \mathbf{0} \mid(u: v) \notin U_{g}\right\} .
$$


We can now put $Q=N \times[0,1] \subset \bar{B}_{\delta_{\Phi}(\varepsilon)}^{4} \times[0,1]$ : this gives the desired isotopy between the internal Milnor subfibration of $g$ defined by $E_{g}^{\text {int }}=\left\{z \in E_{g} \mid(f(z): g(z)) \notin U_{g}\right\}$ and a Milnor subfibration of $f+g$.

The proof of Theorem 1.3 is exactly the same, except that we can put $N=\bar{B}_{\delta_{\Phi}(\varepsilon)}^{4}$.

REMARK. More generally, for two arbitrary distinct directions $\tau$ and $\tau^{\prime}$ in $\mathbb{P}^{1}$, and $U_{\tau, \tau^{\prime}} \subset \mathbb{P}^{1}$ an open contractible neighbourhood of $\tau$ and $\tau^{\prime}$ with smooth boundary containing no point of $\mathbb{P} C_{0}$ Disc $\Phi$ other than $\tau$ and $\tau^{\prime}$, the same proof shows that the two subsets

$$
\begin{aligned}
& E_{\tau}^{\tau, \tau^{\prime}}:=\left\{z \in E_{\tau} \mid(f(z): g(z)) \notin U_{\tau, \tau^{\prime}}\right\}, \\
& E_{\tau^{\prime}}^{\tau, \tau^{\prime}}:=\left\{z \in E_{\tau^{\prime}} \mid(f(z): g(z)) \notin U_{\tau, \tau^{\prime}}\right\}
\end{aligned}
$$

of the total spaces of the Milnor fibrations of $\varphi_{\tau}$ and $\varphi_{\tau^{\prime}}$ define isotopic Milnor subfibrations.

COROllary 4.3. Suppose that $f$ is a non-transverse member of the pencil $\mathscr{P}(\Phi)$ associated with a pair $\Phi=(f, g):\left(\mathbb{C}^{n+1}, \mathbf{0}\right) \rightarrow\left(\mathbb{C}^{2}, \mathbf{0}\right)$ with no blowing up. Then the characteristic polynomials of the top-dimensional homological monodromies of $f$ and a generic deformation $f+t g$ have a common factor, given by the top-dimensional homological monodromy of the internal Milnor subfibration of $f$.

More generally, the homological zeta functions associated with $f$ and $f+t g$ have a common factor given by the internal Milnor subfibration of $f$.

Proof. The second statement is a direct consequence of the properties of the homological zeta function, while the first comes from the inclusions of $H_{n}\left(F_{f}^{\text {int }}, \mathbb{Z}\right)$ in $H_{n}\left(F_{f}, \mathbb{Z}\right)$ and $H_{n}\left(F_{f+t g}, \mathbb{Z}\right)$, and the fact that they commute with the different monodromies.

ExAmple 4.4. The last theorem, together with Example 4.2, shows that, when $\varphi_{\tau}$ is a non-transverse member such that the only component of Disc $\Phi$ tangent at the origin to the $\tau$-directional complex line is this line itself, its Milnor fibration can be embedded in the one of a transverse member $\varphi_{\text {trans }}$. This can be used in the following situation: if the pair $\Phi=(f, g)$ has no blowing up, then this is also the case for the pair $\Phi_{N}:=\left(f, g^{N}\right)$. Now if $f$ is a non-transverse member of $\mathscr{P}(\Phi)$, then for $N$ sufficiently large it will satisfy the preceding hypothesis in $\mathscr{P}\left(\Phi_{N}\right)$, and so its Milnor fibration will be embedded in the one of a transverse member $f_{t}=f+\operatorname{tg}^{N}$.

In particular, by induction on the dimension $s$ of the critical set Crit $f$, we recover the result of Tibăr (see [27]), saying that the Milnor fibration of any germ $f:\left(\mathbb{C}^{n+1}, \mathbf{0}\right) \rightarrow(\mathbb{C}, 0)$ can be embedded in that of a deformation $f+t_{1} \ell_{1}^{N_{1}}+\ldots+t_{s} \ell_{s}^{N_{s}}$ with an isolated singularity at $\mathbf{0}$.

\subsection{A spectral sequence}

Our final result, or more precisely the remark following it, when applied simultaneously to all the non-transverse members (which is possible, thanks to 
Lemma 3.8 with $\boldsymbol{\tau}=\mathbb{P} C_{\mathbf{0}}$ Disc $\Phi \cup\{$ trans $\}$ ), gives a result which is a first (modest) step towards a precise formulation of the following conjectural credo: the generic topology can be built from the atypical ones.

For a $(p+1)$-tuple $\left(\tau_{0}, \ldots, \tau_{p}\right)$ of non-transverse parameters, $F_{\tau_{0}, \ldots, \tau_{p}}^{\mathrm{int}}$ will denote the intersection of the images of the internal Milnor subfibres $F_{\tau_{i}}^{\text {int }}{ }^{p}$ in the Milnor fibre $F_{\text {trans }}$ of a transverse member. These intersections $F_{\tau_{0}, \ldots, \tau_{p}}^{\mathrm{int}}$ are the fibres of Milnor subfibrations of $\varphi_{\text {trans }}$. Each homology group of these subfibres, as for $F_{\text {trans }}$, is then endowed with an endomorphism induced by the monodromy, that is, with a $\mathbb{Z}\left[x, x^{-1}\right]$-module structure. But if the pencil has at least two nontransverse members, one can suppose that the fibre $F_{\text {trans }}$ is covered by the union of all the images of the internal subfibres $F_{\tau}^{\text {int }}$. The Mayer-Vietoris spectral sequence associated with this cover allows one to state the following.

THEOREM 4.5. For every $\mathscr{P}(\Phi)$ associated with a pair $\Phi$ with no blowing up and with at least two non-transverse members, there is a spectral sequence of $\mathbb{Z}\left[x, x^{-1}\right]$-modules, given by

$$
E_{p q}^{1}=\bigoplus_{\substack{\tau_{i_{j}} \in \mathbb{P} C_{0} \text { Disc } \Phi \\ i_{0}<\ldots<i_{p}}} H_{q}\left(F_{\tau_{i_{0}}, \ldots, \tau_{i_{p}}}^{\mathrm{int}}, \mathbb{Z}\right) \Longrightarrow H_{p+q}\left(F_{\text {trans }}, \mathbb{Z}\right) .
$$

4.3. Comparison between the Milnor fibres of a non-transverse member and of the transverse one

We will now determine the 'maximal' (with respect to our method) Milnor subfibre of a non-transverse member $\varphi_{\tau}$ that can be embedded in the Milnor fibre of the transverse member $\varphi_{\text {trans. }}$. It will, of course, contain the internal Milnor subfibre but will not be a priori the fibre of a common subfibration. This subfibre will be, in certain cases, isotopic to the Milnor fibre of the non-transverse member, or to that of the transverse one. There are three types of non-transverse parameters in $\mathbb{P}_{C_{0}}$ Disc $\Phi$ (which were all illustrated in Figure 2): $\tau$ is of Type I if the $\tau$-directional line passing through $\mathbf{0}$ is the only component of Disc $\Phi$ having $\tau$ as tangent direction at $\mathbf{0}$, of Type II if this line is not a component of Disc $\Phi$, and of Type III in the remaining case.

THEOREM 4.6. Let $\varphi_{\tau}$ be a non-transverse member of the pencil associated with a pair with no blowing up. Then one has the following.

(i) If $\tau$ is of Type I, the Milnor fibration of $\varphi_{\tau}$ is isotopic to its internal Milnor subfibration. In particular, it can be embedded in the Milnor fibration of a transverse member.

(ii) If $\tau$ is of Type II, the Milnor fibre $F_{\text {trans }}$ of a transverse member can be embedded in the Milnor fibre $F_{\tau}$ of $\varphi_{\tau}$, and its image contains the internal Milnor subfibre.

(iii) If $\tau$ is of Type III, the Milnor fibre $F_{\text {trans }}$ of a transverse member and the Milnor fibre $F_{\tau}$ of $\varphi_{\tau}$ have isotopic subfibres that contain the internal Milnor subfibre and its isotopic image in $F_{\text {trans }}$ (but are possibly larger).

Proof. We make the same assumptions as in the beginning of the proof of Theorem 1.5, and use the same notation. 
For $y \neq 0$ in $\mathbb{C}$ sufficiently small, consider the set $\widetilde{Q}^{y}$ in $\bar{B}_{\delta_{\Phi}(\varepsilon)}^{4} \times[0,1]$ defined by

$$
\widetilde{Q}^{y}:=\bigcup_{t \in[0,1]} L_{t}^{y} \times\{t\} .
$$

The proof of Proposition 2.4 can then be rephrased to say that for all $r>0$ there is a $y \neq 0$ with $|y|<r$ and a closed submanifold with corners $Q^{y} \subset \widetilde{Q}^{y}$ meeting the product Disc $\Phi \times[0,1]$ transversally. Moreover, the restriction $H^{y}$ of $H$ to $Q^{y}$ (which is simply the projection on the second factor in this case) is a proper submersion of the pair $\left(Q^{y}, Q^{y} \cap(\operatorname{Disc} \Phi \times[0,1])\right)$ on $[0,1]$. Finally, the fibres $Q_{0}^{y}$ and $Q_{1}^{y}$ of this restriction over 0 and 1 are exactly the isotopic marked subdiscs of the horizontal and of the oblique marked discs that are considered in this proposition.

The product $\Phi_{\varepsilon} \times \operatorname{Id}_{[0,1]}$ lifts the isotopies; hence these isotopic marked subdiscs correspond to isotopic Milnor subfibres of the non-transverse member $g$ and of the transverse member $f+g$ respectively. The conclusions then follow from those of Proposition 2.4.

The cases I and II are the most tractable: they show that in certain cases, one of the Milnor fibres we compare is obtained from the other by attaching some handles. This may sometimes be used to determine when a non-transverse parameter is really atypical. For instance, the following holds.

Corollary 4.7. Suppose that $n=1$, or that all the members of the pencil $\mathscr{P}(\Phi)$ with no blowing up have an isolated singularity at $\mathbf{0}$ and that the critical locus of $\Phi$ is of dimension at most 1 . Then the approximation of Theorem 1.3 is sharp: one has $A_{\Phi}=\mathbb{P} C_{0}$ Disc $\Phi$.

Proof. Put $n \geqslant 2$. When a germ $f:\left(\mathbb{C}^{n+1}, \mathbf{0}\right) \rightarrow(\mathbb{C}, 0)$ has an isolated singularity at $\mathbf{0}$, the homotopy type of the Milnor fibre $F_{f}$ is exactly the homotopy type of the universal covering $\left(B_{\varepsilon}^{2 n+2} \backslash V(f)\right)^{\sim}$ (where $(\cdot)^{\sim}$ denotes $(\widetilde{\bullet})$ ) of the complement of the hypersurface germ it defines (this is true more generally whenever $F_{f}$ is simply connected, which is the case when $\operatorname{codim}_{V(f)}$ Crit $f \geqslant 2$ ). It follows that in order to show that the embedded topologies of the hypersurface germs they define are different, it suffices to show that the Milnor fibre of a non-transverse member $\varphi_{\tau}$ of $\mathscr{P}(\Phi)$ has a different homotopy type from that of a transverse member.

But the facts that the critical locus Crit $\Phi$ is a curve, and that $\varphi_{\tau}$ has an isolated singularity, imply that $\tau$ is of Type II and then that its Milnor fibre is obtained from that of a transverse member $\varphi_{\text {trans }}$ by attaching some $n$-handles (see [3, III.3.2] for a more detailed discussion). Their Euler characteristics are then different.

For $n=1$, the same argument is valid for the Type II parameters. But the Type I and Type III parameters are necessarily associated with non-isolated singularities, which in the case of plane curves are known not to have the same topology as isolated ones.

Remark. In association with Parusiński's result [23], this shows that ${ }^{P} C_{0}$ Disc $\Phi$ is in this case exactly the set of parameters $\tau$ where the Milnor number $\mu\left(\varphi_{\tau}\right)$ 'jumps'. 


\section{Examples}

\subsection{Generalized Iomdine-Lê deformations}

Let $f:\left(\mathbb{C}^{n+1}, \mathbf{0}\right) \rightarrow(\mathbb{C}, 0)$ be a complex analytic germ. We call any germ $f_{t}=f+t \ell^{N}$ a generalized Iomdine-Lê deformation of $f$, where $\ell$, a linear form, and $t \in \mathbb{C}$ are both sufficiently general, and $N$ is a positive integer.

REMARK. These deformations have been considered by many authors (see [8, 11, 25]), but only when $N$ is sufficiently large. But without requiring $N$ to be large, these deformations appear naturally in the study of the singularities at infinity of polynomials: if $F: \mathbb{C}^{n+1} \rightarrow \mathbb{C}$ is a polynomial and $\widetilde{F}$ is its homogenization, then $f$ will be the germ of $\widetilde{F}$ in an affine chart of $\mathbb{C}^{n+1}$ at one of its zeros in the hyperplane at infinity $\mathbb{P} \mathbb{C}^{n+1} \backslash \mathbb{C}^{n+1}$, and $g=\ell^{\operatorname{deg} F}$ will be an equation of this hyperplane, to the adequate power. The hypersurface germs $V\left(f_{t}\right)=\left\{z \mid f(z)=-t \ell(z)^{\operatorname{deg} F}\right\}$ then correspond to the fibres $F^{-1}(-t)$ of $F$. One must nevertheless be cautious: it may happen that the genericity condition on $\ell$ is not satisfied in this case (think of the example $\left(y^{2}-x z, x\right)$ seen previously, which corresponds for instance to the polynomial $\left.F(X, Y, Z)=Y^{2}-Z\right)$. However, it is always satisfied in the finite case, that is, when $n=1$.

When the genericity conditions are assumed, the pair $\Phi_{N}=\left(f, \ell^{N}\right)$ has no blowing up for all $N>0$, and these deformations are exactly the generic members of the pencil $\mathscr{P}\left(\Phi_{N}\right)$. We can then use our methods to compare the Milnor fibration of $f$ with that of any such deformation $f_{t}$.

To state the result, we need first to collect a few facts about the polar exponents and the polar filtration (see for instance [10]). To any pair $(f, \ell)$, we can associate a finite increasing sequence $\mathbf{e}(f)=\left\{e_{1}<\ldots<e_{r}\right\}$ of rational numbers greater than 1 , the polar exponents of $f$ with respect to $\ell$. They are the ratios of the first powers that appear in the Puiseux parametrization of the different branches of the discriminant $\operatorname{Disc}(f, \ell)$, which, as we have seen above, is tangent to the vertical axis at $\mathbf{0}$ (this explains why these ratios are greater than 1.) Note that we have chosen a different convention from the one in [10]: our polar exponents are the inverses of the ones of Lê.

One can associate to the sequence $\mathbf{e}(f)$ an increasing filtration of the Milnor fibre of $f$, the polar filtration, which can be supposed compatible with the monodromy: we have

$$
F_{\left.f\right|_{V(\ell)}}=: F_{f}^{0} \subset F_{f}^{e_{1}} \subset \ldots \subset F_{f}^{e_{r}}=F_{f}
$$

with $F_{f}^{e_{i}}:=\left\{\left.z \in F_{f}|| \ell(z)\right|^{e_{i}} \leqslant M|f(z)|\right\} \quad(M \gg 1)$. Furthermore, every piece of this filtration is obtained from the preceding piece by adding some $n$-handles.

Proposition 5.1. To every positive integer $N$ we associate a number $e(N)$ which is the greatest polar exponent less than or equal to $N$, or 0 if $N<e_{1}$. Then the piece $F_{f}^{e(N)}$ of the polar filtration of the Milnor fibre of $f$ can be embedded in the polar filtration of the Iomdine-Lê deformation $f+t \ell^{N}$, and it is the fibre of a common Milnor subfibration.

Proof. It suffices to identify $F_{f}^{e(N)}$ with the internal subfibre $F_{(0: 1)}^{\mathrm{int}}$ of the Milnor fibre of $f$ in the pencil associated with $\Phi_{N}=\left(f, \ell^{N}\right)$. 
REMARK. This result suggests the following heuristic description of the polar filtration: it is formed by the pieces which the Milnor fibration of $f$ shares with the fibration of a Iomdine-Lê deformation $f+t \ell^{N}$, with $N$ increasing.

\subsection{The Malgrange pencil}

Let $\Phi=(f, g):\left(\mathbb{C}^{n+1}, \mathbf{0}\right) \rightarrow\left(\mathbb{C}^{2}, \mathbf{0}\right)$ be defined by

$$
\Phi(z)=\left(\left(z_{0} \ldots z_{n}\right)^{2}, z_{0}^{2 n+4}+\ldots+z_{n}^{2 n+4}\right) .
$$

We call the pencil $\mathscr{P}(\Phi)$ associated with $\Phi$ the Malgrange pencil for the following reason: its generic member is precisely the example Malgrange studied in [18] to show that the bound for the quasi-unipotency given by the Monodromy Theorem is sharp. We will illustrate our methods by computing the Milnor number of this generic member $\varphi_{\text {gen }}=f+g$. See also [1]. For details, see [3].

The Milnor fibres of $f, g$ and $f+g$ will be denoted by $F_{f}, F_{g}$ and $F_{\text {gen }}$, respectively. One has:

$$
F_{f} \simeq(\underbrace{S^{1} \times \ldots \times S^{1}}_{n \text { times }}) \times\{0,1\}, \quad F_{g} \simeq \underbrace{S^{n} \vee \ldots \vee S^{n}}_{(2 n+3)^{n+1} \text { times }} .
$$

The pair $\Phi$ has no blowing up: the hypersurfaces $V(f)$ and $V(g)$ have natural Whitney stratifications, given by

$$
\begin{aligned}
& V(f)=\coprod_{\emptyset \neq I \subset\{0, \ldots, n\}}\left\{z_{i}=0, i \in I ; z_{j} \neq 0, j \notin I\right\}, \\
& V(g)=(V(g) \backslash\{\mathbf{0}\}) \cup\{\mathbf{0}\} .
\end{aligned}
$$

These stratifications are transverse to each other. The induced intersection stratification on $V(\Phi)$ can then be shown to be good.

The critical locus Crit $\Phi$ splits: Crit $\Phi=\operatorname{Crit} f \cup \Gamma$, where $\Gamma$ is the curve given by $z_{i}^{2 n+4}=z_{j}^{2 n+4}$ for all $i$ and $j$. This curve is the union of $(2 n+4)^{n}$ distinct lines. One can show that $\Phi$ has only $A_{1}$ singularities along $\Gamma \backslash\{\boldsymbol{0}\}$.

The discriminant Disc $\Phi$ splits accordingly: $\operatorname{Disc} \Phi=\mathbb{C}(0: 1) \cup \Delta$, with $\Delta=\Phi(\Gamma)$ having $(1: 0)$ as tangent direction at $\mathbf{0}$. Thus one gets $\mathbb{P} C_{0} \operatorname{Disc} \Phi=\{(1: 0),(0: 1)\}$, with $(1: 0)$ of Type II and $(0: 1)$ of Type I. Here, the approximation given by the Theorem 1.3 is sharp (for $(1: 0)$, it is a direct application of Corollary 4.7; for $(0: 1)$ the Milnor fibre of $\varphi_{(0: 1)}=f$ is not a bouquet of $n$-spheres, so $f$ cannot be topologically equivalent to the generic member, which defines an isolated singularity).

The following four assertions connect with each other the homotopy types of $F_{f}, F_{g}, F_{\text {gen }}$ and that of the fibre $F_{\mid}$of $\Phi$ :

(a) the fibre $F_{\text {gen }}$ is homotopy equivalent to the union of the two internal subfibres $F_{f}^{\text {int }}$ and $F_{g}^{\text {int }}$, their intersection being homotopy equivalent to the fibre $F_{\mid}$;

(b) one gets $F_{f}$ from $F_{\mid}$by attaching $(\Gamma, V(f))_{0} n$-handles (the last notation designates the intersection number);

(c) the fibre $F_{f}$ is homotopy equivalent to its internal subfibre $F_{f}^{\text {int }}$;

(d) one gets $F_{g}$ from its internal subfibre $F_{g}^{\text {int }}$ by attaching $(\Gamma, V(g))_{0} n$-handles. 
We then get the following system of equations:

$$
\left\{\begin{aligned}
\chi\left(F_{\text {gen }}\right) & =1+(-1)^{n} \mu_{\text {gen }}=\chi\left(F_{f}^{\text {int }}\right)+\chi\left(F_{g}^{\text {int }}\right)-\chi\left(F_{\mid}\right), \\
\chi\left(F_{f}\right) & =\chi\left(F_{\mid}\right)+(-1)^{n}(\Gamma, V(f))_{0}, \\
\chi\left(F_{f}\right) & =\chi\left(F_{f}^{\text {int }}\right), \\
\chi\left(F_{g}\right) & =\chi\left(F_{g}^{\text {int }}\right)+(-1)^{n}(\Gamma, V(g))_{0} .
\end{aligned}\right.
$$

We know already that $\chi\left(F_{f}\right)=0$ and $\chi\left(F_{g}\right)=1+(-1)^{n}(2 n+3)^{n+1}$. Moreover, since $\Gamma$ is the union of $(2 n+4)^{n}$ lines, none of which is contained in $V(f)$, we get

$$
(\Gamma, V(f))_{\mathbf{0}}=(\operatorname{deg} f)(2 n+4)^{n}=(2 n+2)(2 n+4)^{n},
$$

and, analogously,

So, finally,

$$
(\Gamma, V(g))_{\mathbf{0}}=(\operatorname{deg} g)(2 n+4)^{n}=(2 n+4)^{n+1} .
$$

$$
\begin{aligned}
\mu_{\text {gen }} & =(2 n+2)(2 n+4)^{n}+(2 n+3)^{n+1}-(2 n+4)^{n+1} \\
& =(2 n+3)^{n+1}-2(2 n+4)^{n} .
\end{aligned}
$$

For $n=2$, we find that $\mu_{\text {gen }}=215$, which was noticed by A'Campo in [1].

\section{References}

1. N. A'CAmpo, 'La fonction zêta d'une monodromie', Comment. Math. Helv. 50 (1975) 233-248.

2. J. Briançon, P. Maisonobe and M. Merle, 'Localisation des systèmes différentiels, stratifications de Whitney et condition de Thom', Invent. Math. 117 (1994) 531-550.

3. C. Caubel, 'Sur la topologie d'une famille de pinceaux de germes d'hypersurfaces complexes', doctoral thesis, Université Toulouse III, 1998.

4. C. Caubel, idem, C. R. Acad. Sci. Paris Sér. I 328 (1999) 501-504.

5. A. H. DurfeE, 'Neighbourhoods of algebraic sets', Trans. Amer. Math. Soc. 276 (1983) $517-530$.

6. M. Goreski and R. MacPherson, Stratified Morse theory, Ergebnisse der Mathematik und ihrer Grenzgebiete (3) 14 (Springer, Berlin, 1988).

7. J.-P. Henry, M. Merle and C. Sabbah, 'Sur la condition de Thom stricte pour un morphisme analytique complexe', Ann. Sci. École Norm. Sup. (4) 17 (1984) 227-268.

8. I. N. Iomdin, 'Complex surfaces with a one-dimensional set of singularities', Siberian Math. J. 15 (1974) 748-762.

9. Lê D. T., 'Calcul du nombre de cycles évanouissants d'une hypersurface complexe', Ann. Inst. Fourier 23 (1974) 261-270.

10. LÊ D. T., 'La monodromie n'a pas de points fixes', J. Fac. Sci. Univ. Tokyo Sect. I A 22 (1975) 409-427.

11. LÊ D. T., 'Ensembles analytiques complexes avec lieu singulier de dimension un (d'après I. N. Iomdine)', Séminaire sur les singularités (ed. Lê D. T. et al.), Publications Mathématiques de l'Université Paris VII (Paris, 1980) 87-95.

12. LÊ D. T. and B. Perron, 'Sur la fibre de Milnor d'une singularité isolée en dimension complexe trois', C. R. Acad. Sci. Paris Sér. A 289 (1979) 115-118.

13. LE D. T. and C. P. Ramanujam, 'The invariance of Milnor's number implies the invariance of the topological type', Amer. J. Math. 98 (1976) 67-78.

14. LÊ D. T. and B. TeIssier, 'Cycles évanescents, sections planes et conditions de Whitney. II', Singularities (ed. P. Orlik), Proceedings of Symposia in Pure Mathematics 40, Part 2 (American Mathematical Society, Providence, RI, 1983) 65-103.

15. Lê $\mathrm{D}$. T. and C. Weber, 'Equisingularité dans les pinceaux de germes de courbes planes et $C^{0}$-suffisance', Enseign. Math. (2) 43 (1997) 355-380.

16. F. LoEsEr, 'Fonctions zeta locales d'Igusa à plusieurs variables, intégration dans les fibres, et discriminants', Ann. Sci. École Norm. Sup. (4) 22 (1989) 435-471.

17. E. J. N. LoolJenga, Isolated singular points on complete intersections, London Mathematical Society Lecture Note Series 77 (Cambridge University Press, 1984). 
18. B. Malgrange, 'Letter to the editors', Invent. Math. 20 (1973) 171-172.

19. D. B. Massey, 'The Lê varieties. I', Invent. Math. 99 (1990) 357-376.

20. D. B. Massey, 'The Lê varieties. II', Invent. Math. 104 (1991) 113-148.

21. J. Milnor, Singular points of complex hypersurfaces, Annals of Mathematics Studies 61 (Princeton University Press, 1968).

22. A. NÉmEthi, 'The Milnor fiber and the zeta function of the singularities of type $f=P(h, g)$, Compositio Math. 79 (1991) 63-97.

23. A. PARUSIŃSKI, 'Topological triviality of $\mu$-constant deformations of type $f(x)+\operatorname{tg}(x)$ ', Bull. London Math. Soc. 31 (1999) 686-692.

24. R. Schraumen, 'Series of singularities and their topology', doctoral thesis, Rijksuniversiteit te Utrecht, 1990.

25. D. Siersma, 'The monodromy of a series of hypersurface singularities', Comment. Math. Helv. 65 (1990) 181-197.

26. M. TiвĂR, 'Bouquet decomposition of the Milnor fiber', Topology 35 (1996) 227-241.

27. M. ТıвӑR, 'Embedding non isolated singularities into isolated singularities', Singularities. The Brieskorn Anniversary Volume (ed. V. I. Arnold et al), Progress in Mathematics 162 (Birkhäuser, Basel, 1998) 103-115.

28. J.-P. VANNiER, 'Sur les fibres de Milnor d'une famille à un paramètre de fonctions analytiques à singularité isolée', C. R. Acad. Sci. Paris Ser. I 302 (1986) 229-231.

\author{
Clément Caubel \\ Mathematisches Institut \\ Universität Basel \\ Rheinsprung 21 \\ CH-4051 \\ Basel \\ Switzerland \\ caubel@math.unibas.ch
}

